\title{
Expression of rat I-TAC/CXCL11/SCYA11 during central nervous system inflammation: comparison with other CXCR3 ligands
}

\author{
Shaun R McColl ${ }^{1}$, Surendran Mahalingam ${ }^{1, *, \dagger}$, Maria Staykova ${ }^{2}$, Laurie A Tylaska ${ }^{3}$, \\ Katherine E Fisher ${ }^{4}$, Christine A Strick ${ }^{4}$, Ronald P Gladue ${ }^{3}$, Kuldeep S Neote ${ }^{4}$ and \\ David O Willenborg ${ }^{2}$
}

\begin{abstract}
${ }^{1}$ School of Molecular \& Biomedical Science, Adelaide University, Adelaide, South Australia, Australia; ${ }^{2}$ Neurosciences Research Unit, The Canberra Hospital, Canberra, Australian Capital Territory, Australia; ${ }^{3}$ Department of Immunology and ${ }^{4}$ Department of Molecular Sciences, Pfizer Central Research, Groton, CT, USA
\end{abstract}

\begin{abstract}
The chemokines are a large gene superfamily with critical roles in development and immunity. The chemokine receptor CXCR3 appears to play a major role in the trafficking of activated Th1 lymphocytes. There are at least three major ligands for CXCR3: mig/CXCL9, IP-10/CXCL10 and I-TAC/CXCL11, and of these three ligands, CXCL11 is the least well-characterized. In this study, we have cloned a rat ortholog of CXCL11, evaluated its function, and examined its expression in the Th-1-mediated disease, experimental autoimmune encephalomyelitis (EAE) in the rat. Based on its predicted primary amino-acid sequence, rat I-TAC/CXCL11 was synthesized and shown to induce chemotaxis of activated rat $\mathrm{T}$ lymphocytes in vitro and the in vivo migration of T lymphocytes when injected into the skin. I-TAC/CXCL11 expression, as determined by RT-PCR, increased in lymph node and spinal cord tissue collected from rats in which EAE had been actively induced, and in spinal cord tissue from rats in which EAE had been passively induced. The kinetics of expression were similar to that of CXCR3 and IP-10/CXCL10, although expression of both CXCR3 and IP-10/CXCL10 was more intense than that of I-TAC/CXCL11 and increased more rapidly in both lymph nodes and the spinal cord. Only minor levels of expression of the related chemokine mig/CXCL9 were observed. Immunohistochemistry revealed that the major cellular source of I-TAC/CXCL11 in the central nervous system (CNS) during EAE is likely to be the astrocyte. Together, these data indicate that I-TAC/CXCL11 is expressed in the CNS during the clinical phase of EAE. However, the observation that I-TAC/CXCL11 is expressed after receptor expression is detected suggests that it is not essential for the initial migration of CXCR3-bearing cells into the CNS.

Laboratory Investigation (2004) 84, 1418-1429, advance online publication, 23 August 2004; doi:10.1038/labinvest.3700155
\end{abstract}

Keywords: chemokine; inflammation; EAE; autoimmunity; CXCL11

Cell migration plays a major role in a wide range of biological functions. One such function includes lymphocyte trafficking, which is critical for protective immunity, but when dysregulated, may also drive autoimmunity. ${ }^{1}$ At the molecular level, the process of T-lymphocyte migration into lymph nodes and peripheral tissues follows a multistep

Correspondence: Dr SR McColl, BSc (Hons), PhD, Chemokine Biology, Department of Molecular Biosciences, Adelaide University, Adelaide, South Australia 5005, Australia.

E-mail: shaun.mccoll@adelaide.edu.au.

${ }^{*}$ Current address: Department of Biological Sciences, University of Wollongong, Wollongong, NSW, Australia.

"Recipient of the Peter Doherty Fellowship from the NH\&MRC Australia.

Received 24 September 2003; revised 5 March 2004; accepted 9 March 2004; published online 23 August 2004 extravasation paradigm mediated by several classes of molecules including selectins, integrins, cell adhesion molecules and various members of the chemokine gene superfamily. ${ }^{2}$

The chemokines are a family of over 40 (generally) small, secreted proteins that mediate chemotaxis and induce various functional changes in subsets of leukocytes in vitro. ${ }^{3-5}$ They are produced by a wide variety of cells, of both haematopoietic and nonhaematopoietic origin, and have been shown to play a critical role in the migration and activation of leukocytes in vivo. On the basis of a distinctive cysteine-containing amino-acid motif present in the predicted primary amino-acid sequence, the chemokines can be divided into two major subfamilies, $\mathrm{C}-\mathrm{X}-\mathrm{C}(\alpha)$ and $\mathrm{C}-\mathrm{C}(\beta)$, and two minor subfamilies, $\mathrm{C}(\gamma)$ and $\mathrm{C}-\mathrm{X}_{3}-\mathrm{C}(\delta)$. Members of the $\mathrm{C}-\mathrm{C}$ subfamily, 
which presently contains the majority of the known chemokines, all demonstrate juxtaposition of the first pair of cysteines within the cysteine signature, while the $\mathrm{C}-\mathrm{X}-\mathrm{C}$ and $\mathrm{C}-\mathrm{X}_{3}-\mathrm{C}$ subfamily members contain one or three intervening amino acids between these two cysteines, respectively. The sole member of the $\mathrm{C}$ subfamily is missing the first and third cysteines within the motif. ${ }^{3-5}$

While chemokines play a central role in the regulation of normal immune responses by activating/recruiting leukocytes to specific sites, ${ }^{4,6,7}$ they have been implicated in a range of pathologies including cancer, allergic and inflammatory disorders as well as in infectious diseases such as AIDS. ${ }^{8-11}$ Since chemokines could be important targets for therapeutic interventions in a range of diseases, and the rat is an important species for evaluating therapeutic potential, it is important to clone and characterize rat chemokines.

Of the CXC chemokines, the non-ELR-containing chemokines I-TAC/CXCL11 (The chemokine nomenclature used in this article adheres to the new nomenclature recently described by Zlotnik and Yoshie. ${ }^{12}$ ), ${ }^{13}$ IP-10/CXCL10 ${ }^{14}$ and mig/CXCL9 ${ }^{15}$ bind to a receptor known as CXCR3, ${ }^{16}$ that has been implicated in Th1-dependent inflammation ${ }^{17,18}$ this receptor appears to be expressed at high levels on activated Th1 lymphocytes ${ }^{19,20}$ and CXCR3expressing $\mathrm{T}$ cells have been observed in autoimmune pathologies including arthritis, psoriasis and multiple sclerosis. ${ }^{21-25}$ Evidence documenting the expression patterns of CXCL10 and CXCL9 are abundant in the literature, whereas information regarding CXCL11 expression and function, particularly in vivo, are scarce. However, one of the major common features of expression of these chemokines is their upregulation by IFN $\gamma$, adding further support for a role in Th-1-dependent inflammation. ${ }^{13,26}$ Owing to the potential importance of understanding the pathobiology of the CXCR3/ligand system, we have cloned the rat ortholog of CXCL11 in order to facilitate the study of its biological function in vivo. To this end, we have characterized the activity of this chemokine and have examined its expression in EAE, a Th1-dependent inflammatory disease of the central nervous system (CNS) in the rat. We now report that increased expression of CXCL11 in vivo occurs in the draining lymph nodes during induction of EAE, and in the spinal cord prior to clinical disease onset but only after the appearance of CXCR3, suggesting that it is not involved in the initial recruitment of activated CXCR3 ${ }^{+} \mathrm{T}$ cells into the CNS.

\section{Materials and methods}

\section{Cloning of Rat CXCL11 and CXCR3}

Degenerate primers were designed against conserved regions of the amino-acid sequence of human CXCL11. ${ }^{13}$ Primers were fully degenerate only through the first five amino acids and were pooled out in order to limit the degeneracy in any given pool to 64 -fold. The $5^{\prime}$ primers were designed against the amino-acid sequence RCLCI, at positions 29-33, and were split into three pools, while the $3^{\prime}$ primers were designed against the amino-acid sequence CLNPKSK, at positions $74-80$, and were also split into three pools. Primers were as follows:

\section{5' Primer pools: \\ 1. AGGGGCMGNTGYCTRTGYAT \\ 2. AGGGGCMGNTGYCTYTGYAT \\ 3. AGGGGCMGNTGYTTRTGYAT}

\section{3' Primer pools:}

\section{CTTGCTYTTNGGRTTYAGRCA \\ 5. CTTGCTYTTNGGRTTRAGRCA \\ 6. CTTGCTYTTNGGRTTYAARCA}

Primer pools were mixed in all possible combinations to generate nine sets of primer pairs. Each pair was run with oligo dT-primed first strand cDNA as template. The template cDNA was generated from primary rat astrocyte RNA stimulated for $12 \mathrm{~h}$ with $\mathrm{TNF} \alpha$, IFN $\gamma$ and IL-1 $\beta$. Polymerase chain reaction (PCR) conditions were five cycles of denaturation at $94^{\circ} \mathrm{C}$ for $1 \mathrm{~min}$, annealing at $37^{\circ} \mathrm{C}$ for $2 \mathrm{~min}$ and extension at $72^{\circ} \mathrm{C}$ for 2 min followed by 30 more cycles where the annealing temperature was increased to $50^{\circ} \mathrm{C}$. PCR products were acrylamide gelpurified and subcloned into pCR 2.1 (Invitrogen, Carlsbad, CA, USA) for automated sequence analysis using an Applied Biosystems Sequencer (model 373; Applied Biosystems, Inc. Foster City, CA, USA). Sequences were analysed using the Basic Local Alignment Search Tool (BLAST, National Center for Biotechnology Information, Bethesda, $\mathrm{MD}$, $\mathrm{USA}^{27}$ ), to identify those related to human CXCL11. Sequences homologous to human CXCL11 obtained from a series of products from independent PCR reactions and independently generated cDNA samples were then compared to obtain a consensus sequence for this region of rat CXCL11.

Sequence information obtained from degenerate PCR described above was used to design the following primers for $5^{\prime}$ RACE: 5'-AATGGCAG CAATCAAGGAAG- $3^{\prime}$ and $5^{\prime}$-GAGTAACGGCTGTGA CAAAG-3' along with a gene-specific complementary DNA (cDNA) amplification primer: $5^{\prime}$-AC CTTTGTCCTTTATGAG-3'. The same sequence data were also used to design $3^{\prime}$ RACE primers: $5^{\prime}$ CTTTGTCACAGCCGTTACTC- $3^{\prime}$ and $5^{\prime}$-TTGATTG CTATTTTGAC- $3^{\prime} .5^{\prime}$ RACE products were generated using the $5^{\prime}$ RACE System for Amplification of cDNA Ends (GIBCO-BRL) according to the manufacturer's instructions. Both oligo dT- and genespecific primers were used to generate cDNA samples from stimulated rat astrocyte RNA for use as templates. $3^{\prime}$ RACE products were generated using the $3^{\prime}$ RACE System (Gibco-BRL) again with 
stimulated rat astrocyte RNA as template. Amplification products were gel-purified and subcloned into pCR 2.1 for automated sequencing as described above. Consensus sequences were determined by comparison of data from several products obtained from several independent reactions from each RACE system. The entire rat CXCL11 coding sequence was then PCR amplified using the following primers: $5^{\prime}$ CTGCTCTCTGCGAAGAAA- $3^{\prime}$ and $5^{\prime}$-GTCAGCT TCTTGGCACAG- $3^{\prime}$. The PCR product was ligated directly into pCR2.1 and sequence confirmed as described above.

The predicted mature rat CXCL11 amino-acid sequence was synthesized as previously described. ${ }^{28}$ A partial rat CXCR3 cDNA was amplified by degenerate RT-PCR using primers based on published human and mouse CXCR3 Genbank entries (NP_001495 and O88410, respectively). Sequencing of the amplified PCR product revealed that it was highly homologous to both mouse and human CXCR3.

\section{Synthesis of RatCXCL11 and Production of Anti-RatCXCL11 Antibodies}

RatCXCL11 was synthesized using automated FMOC-based solid-phase peptide synthesis on an ABI model 431A solid-phase peptide synthesizer (software version Synthassist ${ }^{\mathrm{TM}}$ 2.0) retrofitted with deprotection monitoring as previously described..$^{13}$ Correct electrospray (PE-Sciex API100) masses were obtained for the final products. Rabbit antiratCXCL11 polyclonal antibodies were produced by Cambridge Research Biochemicals (Northwich, UK) and supplied as a protein A-sepharose IgG fraction.

\section{Rats}

Female Lewis rats aged 7-14 weeks were used throughout the EAE study. These were bred at the Animal Breeding Establishment of the Australian National University under specific pathogen-free conditions. They were housed in conventional mouse rooms and given food and water ad libitum.

\section{In Vitro Chemotaxis}

Spleen and lymph node cells were collected from rats. Cells were suspended in RPMI media supplemented with $10 \mathrm{mM}$ HEPES buffer, $100 \mathrm{U} / \mathrm{ml}$ penicillin/streptomycin, $0.1 \mathrm{mM}$ nonessential amino acids, $2 \mathrm{mM}$ L-glutamine, and $0.05 \mathrm{mM}$ 2-mercaptoethanol. All components were obtained from Gibco BRL (Rockville, MD, USA). Cells were incubated in RPMI medium either in the presence of $10 \mu \mathrm{g} / \mathrm{ml}$ of Con A (Sigma) or $2 \mu \mathrm{g} / \mathrm{ml}$ PHA (Lidetechnologies, Grand island, NY, USA) for 4 days, or $400 \mathrm{U} / \mathrm{ml}$ IL-2 (BD Biosciences, Bedford,
MA, USA) for 6 days. Rat lymphocyte chemotaxis in response to rat and human CXCL11 was measured using a 48-well Boyden chamber (Neuro Probe, Inc., Cabin John, MD, USA) as previously described. ${ }^{13}$ In brief, agonists were diluted in RPMI medium containing $0.1 \%$ BSA and added to the bottom wells of a 48-well chemotaxis chamber. Cells were resuspended in RPMI/BSA and $10^{5}$ cells were added to the top wells of the chamber. A $5 \mu \mathrm{m}$ PVP-free filter coated on the bottom with $10 \mu \mathrm{g} / \mathrm{ml}$ of human type IV collagen was used. Chambers were incubated for $60 \mathrm{~min}$ in a $5 \% \mathrm{CO}_{2}$-humidified incubator at $37^{\circ} \mathrm{C}$. After the incubation period, the filters were stained with DiffQuik and the number of lymphocytes migrating to the lower surface was counted in six randomly chosen high-powered fields.

\section{In Vivo Lymphocyte Migration Assay}

Single cell suspensions of Lewis rat splenocytes were cultured with $2 \mu \mathrm{g} / \mathrm{ml}$ concanavalin A (ConA). On day 4, the $\mathrm{T}$ lymphoblasts were isolated on a Ficoll density gradient $(d=1.077)$. Naïve Lewis rats were given $9 \times 10^{6}$ cells intravenously (i.v.), and 30 or $300 \mathrm{ng}$ of synthetic rat CXCL11 in $25 \mu \mathrm{l}$, or the equivalent volume of phosphate-buffered saline (PBS), were injected intradermally into discrete sites on the skin on the dorsum of the same rat. After $4 \mathrm{~h}$, the skin around the injection sites was collected and processed for histology.

\section{Induction of Active EAE}

Myelin basic protein (MBP) was purified from frozen guinea-pig spinal cord according to the method of Eylar et al. ${ }^{20}$ GP-MBP in saline was emulsified in an equal volume of incomplete Freund's adjuvant containing heat-killed Mycobacterium butyricum $(4 \mathrm{mg} / \mathrm{ml})$. Rats were anaesthetized with isofluorane prior to immunization with $100 \mu \mathrm{l}$ of emulsion to each hind footpad. Total dose received for each immunization per rat was $25 \mu \mathrm{g}$ of GP-MBP and $400 \mu \mathrm{g}$ M. butyricum.

\section{Passive Transfer of EAE}

Single cell suspensions were prepared from spleens of donor rats immunized 10 days previously with $50 \mu \mathrm{g}$ MBP-CFA as described above. Cells were cultured at $2 \times 10^{6}$ cells $/ \mathrm{ml}$ in RPMI containing $5 \%$ FCS, $5 \times 10^{-5} \mathrm{M} 2 \mathrm{ME}, 2 \mathrm{mM}$ L-glutamine, antibiotics and $2 \mu \mathrm{g} / \mathrm{ml}$ ConA. Cultures were maintained at $37^{\circ} \mathrm{C}$ in an atmosphere of $5 \% \mathrm{CO}_{2}$ for $96 \mathrm{~h}$, harvested, washed and viable cells transferred i.v. at a dose of $4 \times 10^{7}$ cells/recipient. 


\section{Evaluation of Clinical Signs}

Rats were examined on a daily basis beginning from day 8 after immunization and day 3 after passive transfer. Clinical disease severity was assessed and scored as previously described ${ }^{17}$ using a scale from 1 to 5 as follows: 0 , asymptomatic; 1 , flaccid distal half of tail; 2, entire tail flaccid; 3, ataxia, difficulty in righting; 4, hindlimb weakness; 5, hindlimb paralysis.

\section{RT-PCR of Chemokines and Chemokine Receptors}

Total RNA from spinal cord tissue collected at different times after injection of either incomplete Freund's adjuvant or complete Freund's adjuvant were purified essentially as previously described. ${ }^{29,30}$ The primer sequences are as follows: ratCXCL11f, gccttggctgtgatattgtg, ratCXCL11r, agt gaggaaagcttgtcctg-gaggctg; ratCXCL10f, ccgcgcctatc gccaatgagctgcgc, ratCXCL10r, cttggggacaccttttagcatc ttttgg; muCXCL9f, gaactcagctctgccatgaa, muCXCL9r, ttgccgagtccggatctagg; CXCR3f, tcatcttcctgtcagccagc, CXCR3r, caccaccaccaccaccacta: $\beta$-actinf, gcgac gaggcccaga-gcaagagaggc; $\beta$-actinr, gctaggagccaggg cagtaatctcc. The sequence of PCR amplification was one cycle of denaturation at $95^{\circ} \mathrm{C}$ for $2 \mathrm{~min}$, followed by annealing at $56^{\circ} \mathrm{C}$ for $30 \mathrm{~s}$ and extension at $72^{\circ} \mathrm{C}$ for $1 \mathrm{~min}$. This cycle was followed by $30 \mathrm{~s}$ at $95^{\circ} \mathrm{C}, 30 \mathrm{~s}$ at $56^{\circ} \mathrm{C}$, and $1 \mathrm{~min}$ at $72^{\circ} \mathrm{C}$, repeated 33 times. Amplification of all cDNAs was in the linear phase under these conditions. The samples were migrated on a $1 \%$ agarose gel, and Southern blots were performed using PCR-amplified cDNA products as probes to reveal the bands of interest.

\section{MBP-Specific T-Cell Lines}

MBP-specific $\mathrm{T}$ cell lines were established from lymph nodes of Lewis rats immunized 10 days earlier with MBP-CFA. ${ }^{31}$ The cells were propagated by successive rounds of 3 days of stimulation with $10 \mu \mathrm{g} / \mathrm{ml}$ of antigen in the presence of mitomycin Ctreated syngeneic splenocytes as a source of APCs. The $\mathrm{T}$ lymphoblasts were isolated on a Ficoll density gradient and incubated for 10 days in IL-2containing medium. The T-cell lines were used after the fourth round of restimulation during the first 5 days of propagation in IL-2-containing medium.

\section{Immunohistochemistry}

Serial paraffin sections of lumbar spinal cord of Lewis rats with clinical EAE were subjected to antigen retrieval. Endogenous peroxidase activity and nonspecific antibody binding were reduced by incubation with Peroxide block and Power block (InnoGenex Immunohistochemistry Kit, San Remon, CA, USA). The following antibodies were used: mouse anti-rat ED1-biotin (Serotec, Oxford, Eng- land), rabbit anti-glial fibrillary acidic protein (GFAP) (Chemicon International, Temecula, CA, USA) and rabbit anti-rat I-TAC IgG (Cambridge Research Biochemicals, Northwich, UK). The choice of secondary antibodies and the developing procedure were carried out according to the manufacturer's instructions. The sections were counterstained with Mayer's haematoxylin and mounted in SuperMount (Sapphire Bioscience, Australia).

\section{Results}

\section{Cloning of the Rat Ortholog of CXCL11}

A partial rat CXCL11 cDNA was cloned from an unligated cDNA library prepared from IFN $\gamma$-, IL-1 $\beta$ and $\mathrm{TNF} \alpha$-stimulated primary astrocytes, using degenerate oligonucleotides and the PCR. The degenerate oligonucleotides used encompassed conserved regions between the known non-ELR CXC chemokines (see Materials and methods). Sequencing of several PCR clones reveal one with high homology to human CXCL11 (data not shown). This sequence was used to clone the full-length cDNA by $5^{\prime}$ and $3^{\prime}$ RACE-PCR. The deduced nucleotide and amino-acid sequences of rat CXCL11 are shown in Figure 1a. In comparison to known non-ELR CXC chemokines, rat CXCL11 is closest to the human and mouse CXCL11, followed by human and mouse CXCL9 and CXCL10 (Figure 1b,c).

\section{Biological Activity of CXCL11 In Vitro}

Rat CXCL11 was chemically synthesized as previously described ${ }^{28}$ and tested for activity in vitro. The synthetic protein was found to mobilize intracellular calcium in human CXCR3-expressing cell lines with similar potency to human CXCL11, and to heterologously cross-desensitize the receptor to human CXCL10 (data not shown). In order to evaluate the chemotactic activity of rat CXCL11, rat lymphocytes were isolated from peripheral lymph nodes and spleen and incubated in vitro with either concanavalin A, interleukin 2 or phytohaemagglutinin. The ability of rat CXCL11 to induce chemotaxis of these cells was then investigated using the modified Boyden chamber technique (Figure 2). For comparison, human CXCL11 was also used in these experiments. Rat CXCL11 dose-dependently stimulated chemotaxis of all of the preparations of lymphocytes, with maximal chemotaxis being evident at concentrations between 10 and $100 \mathrm{nM}$. Human CXCL11 was generally more potent.

\section{In Vivo Migration of Activated T-Cells Induced by CXCL11}

The ability of synthetic rat CXCL11 to induce migration of $\mathrm{T}$ lymphocytes in vivo was investigated. ConA-activated lymphocytes were injected 


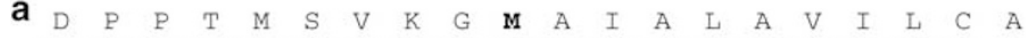
GGATCCGCCGACCATGAGTGTGAAGGGCATGGCTATAGCCTTGGCTGTGATATTGTGTGC

$\downarrow$

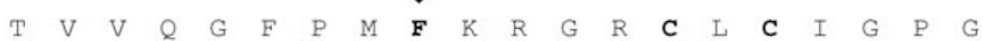
TACAGTTGTTCAAGGCTTCCCCATGTTCAAAAGAGGACGCTGTCTTTGCATAGGCCCTGG

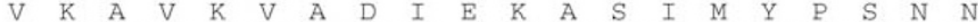
GGTAAAAGCAGTGAAAGTGGCAGATATTGAGAAAGCCTCCATAATGTACCCAAGTAACAA

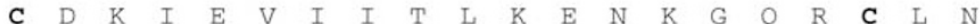
CTGTGACAAAATAGAAGTGATTATTACCCTGAAAGAAAATAAAGGACAACGATGCCTAAA

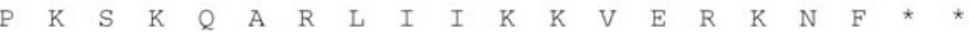
TCCCAAATCGAAGCAAGCAAGGCTTATAATCAAAAAAGTTGAAAGAAAGAATTTTTAATG

P L $\quad$ E

ACCGCTCGAG

b

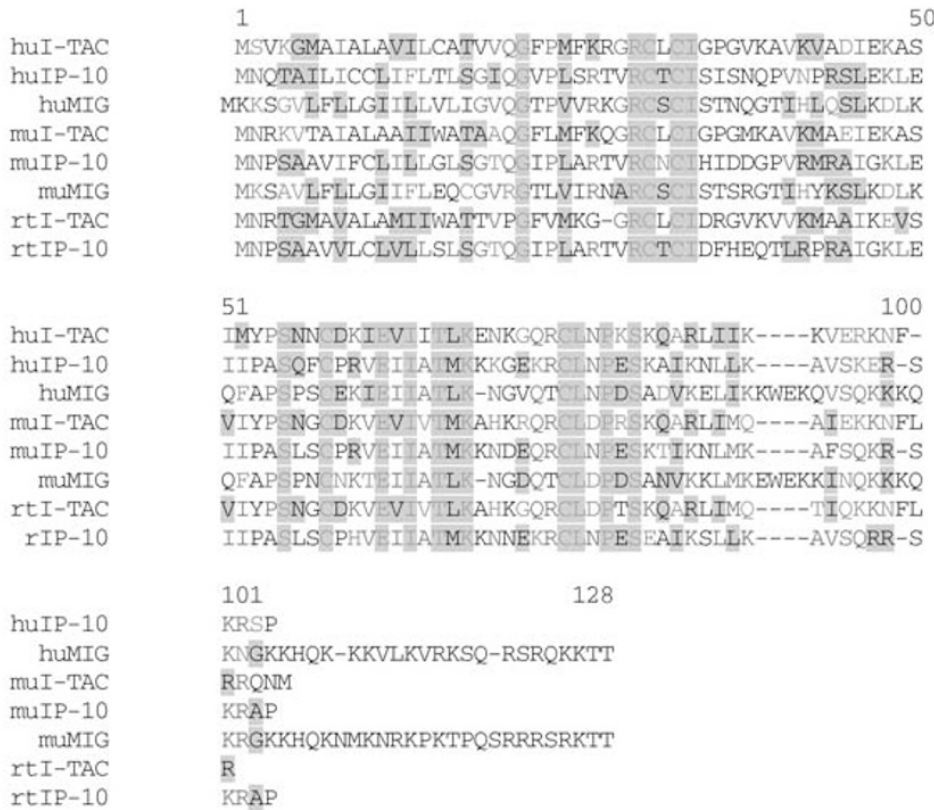

C

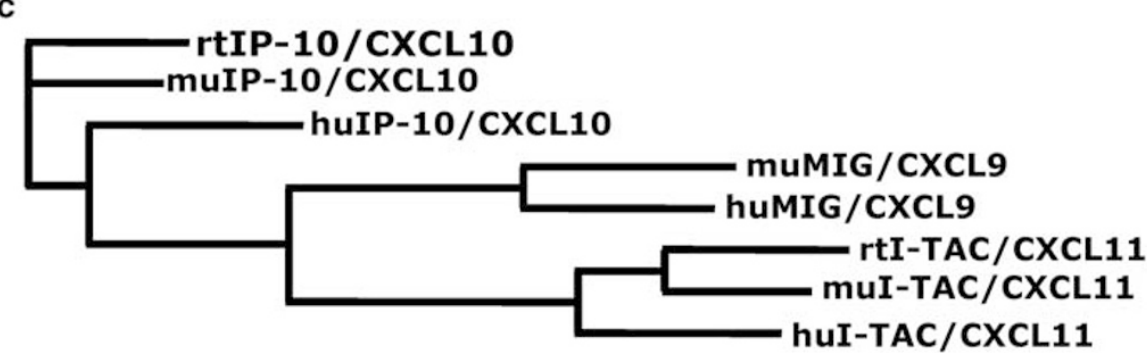

Figure 1 Nucleotide, amino-acid sequence and alignment of rat CXCL11. Rat CXCL11 was cloned as described in Materials and methods. (a) Nucleotide and amino-acid sequence of rat CXCL11. The arrow depicts the predicted amino (N)-terminal amino acid of the mature peptide. (b) Clustal analysis showing alignment of rat, human and mouse CXCR3 ligands. Note that sequence for rat CXCL9 is not currently available. (c) Dendogram showing sequence similarity between CXCR3 ligands across these three species. 


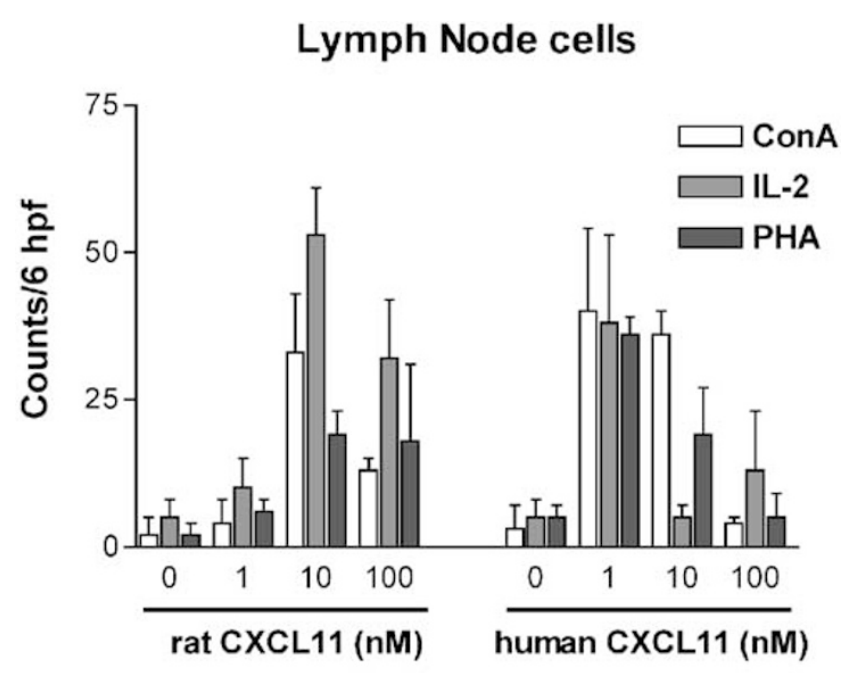

Spleen cells

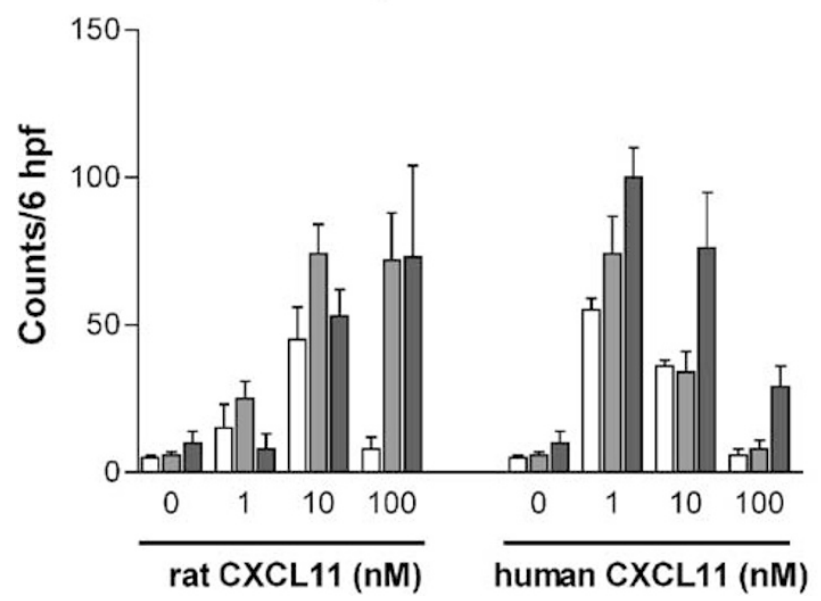

Figure 2 Chemotaxis of rat $\mathrm{T}$ lymphocytes in response to CXCL11. T lymphocytes were isolated from the lymph nodes and spleens and cultured in the presence of either ConA, IL-2 or PHA. The cells were then subjected to modified Boyden chamber chemotaxis assays as described in Material and methods. Data are expressed as mean \pm s.d. of at least $n=10$ values.

intravenously and immediately following this, PBS or rat CXCL11 was injected intradermally into discrete areas on the dorsum of the rat (Figure 3a and b). After $4 \mathrm{~h}$, skin biopsies were collected from the site of injection, sections were prepared and stained with haematoxylin and eosin. Mononuclear cells were conspicuous in the blood vessels, attached to the luminal surface of the endothelial cells, in the perivascular space and in the superficial and deep cutaneous tissue following injection of rat CXCL11. In contrast, few mononuclear cells were observed in the control tissues.

\section{Expression of CXCL11, CXCL10, CXCL9 and CXCR3 in Encephalitogenic T-Cell Lines}

The expression of CXCL11 and CXCR3 in Th1 lymphocytes that are specific for $\mathrm{MBP}$ and that
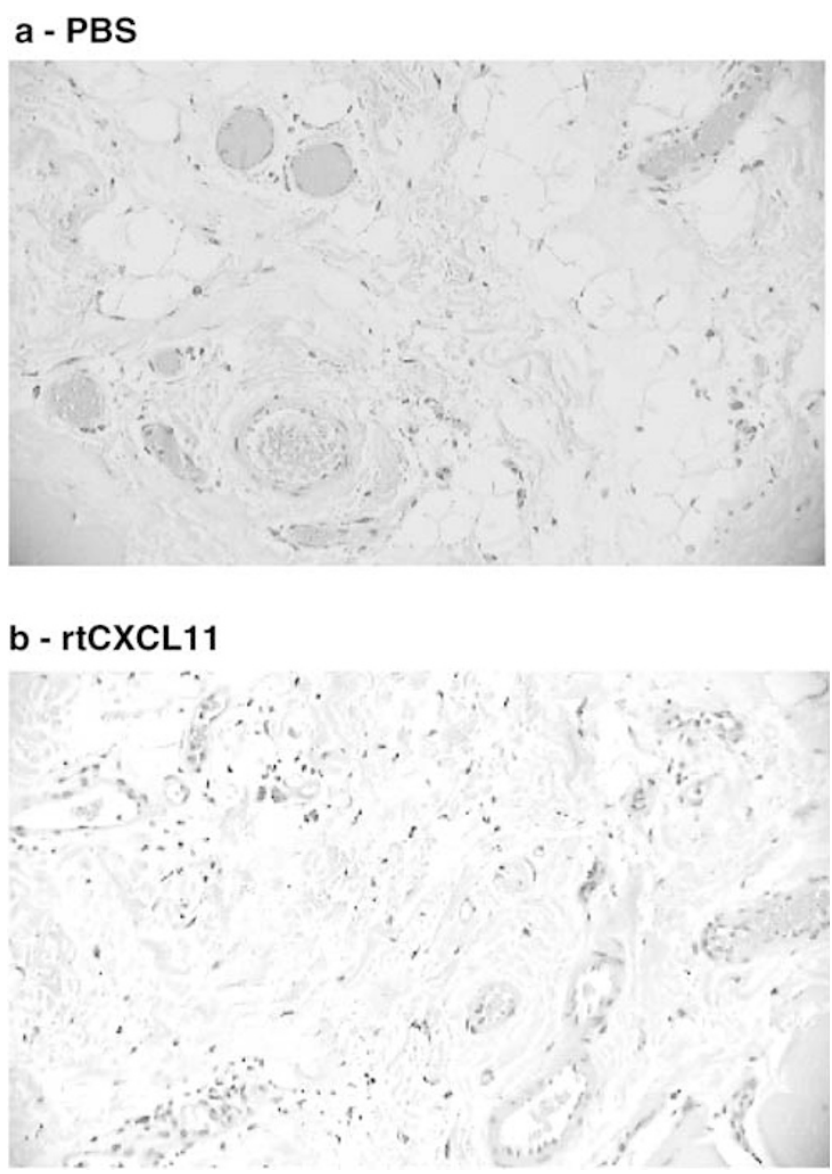

Figure 3 Migration of ConA-activated T lymphocytes in vivo in response to CXCL11. ConA-activated lymphocytes were injected intravenously followed by PBS (a) or 300 ng rat CXCL11 (b) injected into discrete areas on the dorsum of the rat. After $4 \mathrm{~h}$, skin biopsies were collected from the sites of injection, and sections were prepared and stained with haematoxylin and eosin. Representative of three experiments performed with similar results.

induce EAE when injected i.v. into naïve recipients was examined. As there is no quantitative ELISA available for rat CXCL11, expression was determined by RT-PCR. None of the T-cell lines tested following antigen restimulation contained detectable levels of CXCL11, however, the cells expressed CXCR3 messenger RNA (mRNA) (Figure 4). In addition, these cells did not express detectable levels of CXCL10 or CXCL9. These data illustrate two important points. First, that antigen-specific $\mathrm{T}$ lymphocytes are not likely to be a major source of CXCL11, CXCL10 or CXCL9, and second that encephalitogenic $\mathrm{T}$ lymphocytes express CXCR3 mRNA.

\section{Expression of mRNA for CXCL11 and Related Molecules During Active EAE}

Rats were immunized to induce EAE and monitored for induction of disease. For comparison with 
mRNA data, the clinical course of actively induced disease for a representative experiment $(n=12)$ is shown in Figure 5a. RT-PCRs to amplify rat CXCL11,

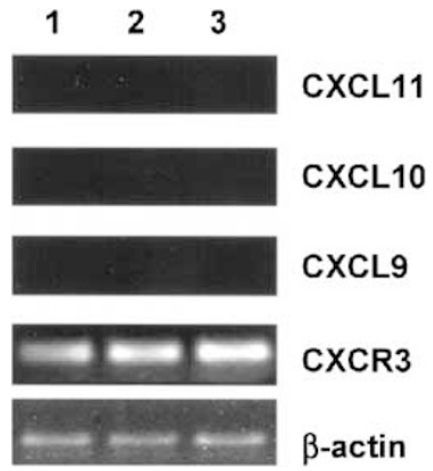

Figure 4 Assessment of expression of CXCR3 and its ligands in encephalitogenic T cell lines. MBP-specific CD4 ${ }^{+}$Th1 T cell lines were established as described in Materials and methods. They were assessed on day 5 of propagation in IL-2-containing medium following restimulation with MBP. Total RNA was isolated and reverse-transcribed. The resultant cDNA was used as a template for PCR using oligonucleotide primers designed to amplify CXCL11, CXCL10, CXCR3 or CXCL9. The data in this figure are representative of three experiments conducted with similar results. the related chemokines CXCL10 and CXCL9 and their receptor CXCR3, were performed on cDNA prepared from either the draining lymph nodes (popliteal) or spinal cords collected at various time points postimmunization following whole body perfusion with $50-100 \mathrm{ml}$ cold saline.

Immunization with MBP-CFA for active EAE led to an increase in the level of CXCL11 expression in the draining lymph nodes (Figure 5b). A low level of CXCL11 expression was detected in the draining lymph nodes as early as day 2 postimmunization with MBP-CFA with maximal level being observed on day 12 postimmunization. CXCR3 expression was also detected on day 2 postimmunization with MBP-CFA in lymph node tissue. Expression of CXCL10 and CXCL9 was also observed on day 2 postimmunization with MBP-CFA, with expression of CXCL10 being the strongest and peaking at around day 8 postimmunization. As expected, the increase in the level of expression of these four genes was not specific to MBP, as similar increases were observed in lymph nodes taken from animals that had been immunized with CFA alone (data not shown).

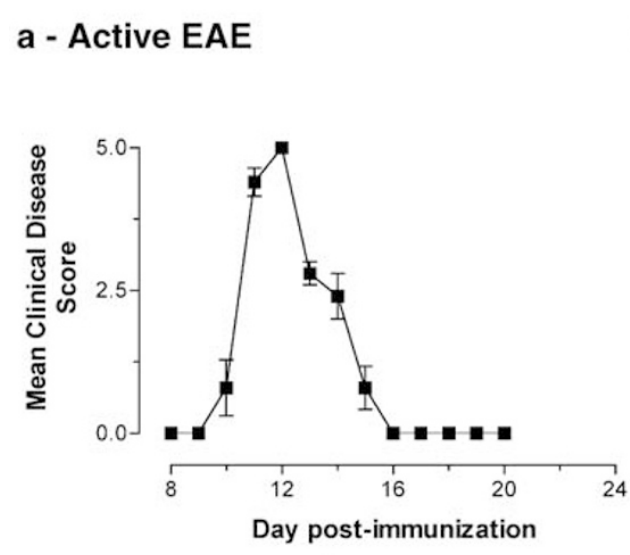

C

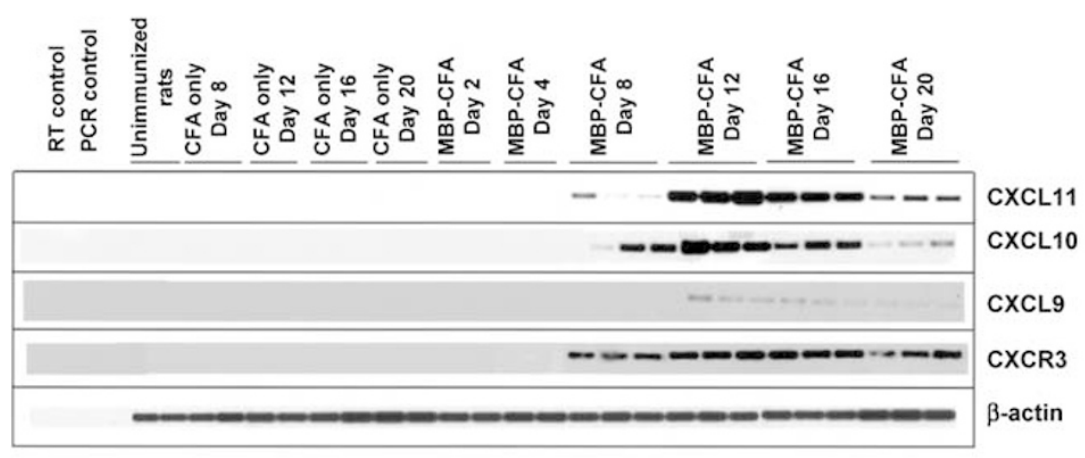

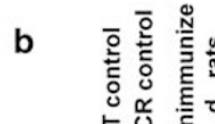
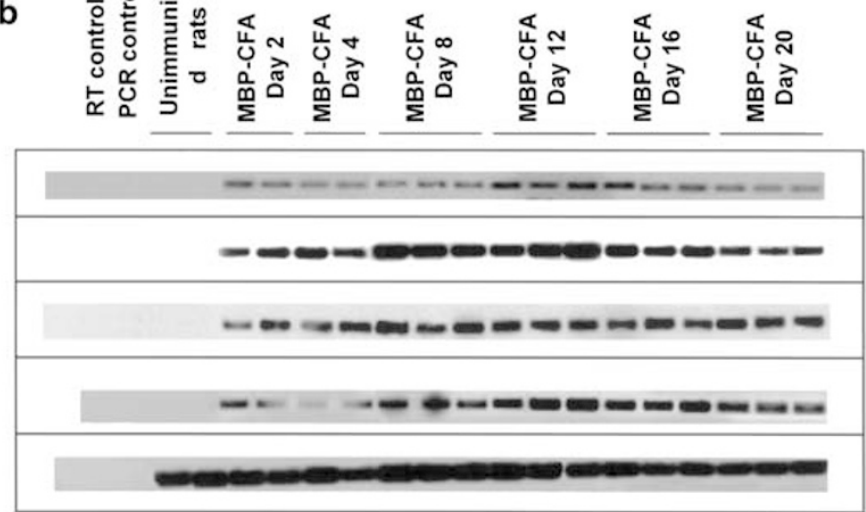

CXCL11

CXCL10

CXCL9

CXCR3

$\beta$-actin d
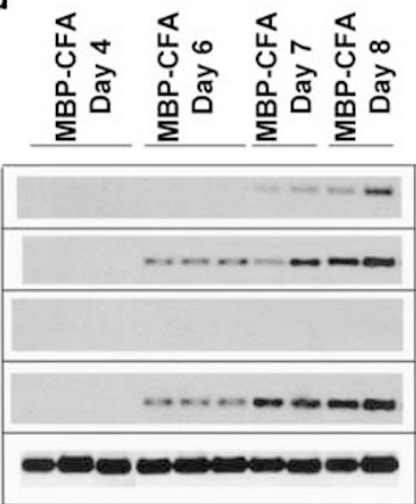

CXCL11

CXCL10

CXCL9

CXCR3

$\beta$-actin

Figure 5 Expression of CXCL11 mRNA in lymph node and spinal cord tissues during active EAE. (a) Rats were immunized with MBPCFA as described in Materials and methods and monitored for clinical signs of EAE. At various time point postimmunization, the animals were killed, perfused and the popliteal lymph nodes and spinal cords collected. Total RNA was purified from the popliteal lymph nodes (b) and the spinal cord (c and d), and reverse-transcribed. The resultant cDNA was used as a template for PCR using oligonucleotide primers designed to amplify CXCL11, CXCL10, CXCR3 or CXCL9. As a control for starting levels of template, $\beta$-actin primers were used. The data points in this figure (ie each band) are from individual rats from the same experiment. They are representative of three experiments conducted with similar results. 
In spinal cord tissue, a weak and variable increase in CXCL11 expression was observed on day 8 postimmunization with MBP-CFA, with maximal levels being observed on day 12 postimmunization (Figure 5c). Strong expression of CXCR3 was observed in the spinal cord from day 8 postimmunization with MBP-CFA with a further increase over the next two time points. Both CXCL11 and CXCR3 remained prominently expressed at day 20 when all animals had recovered from disease. CXCL10 was more strongly expressed than CXCL11 at day 8 but had decreased significantly by recovery at day 20 . CXCL9 expression was only detected at a low level regardless of the time point examined. In contrast to the observations made in the draining lymph nodes, expression of all four genes was dependent on MBP in that expression was not observed in spinal cords taken from animals that had been immunized with PBS-CFA only (Figure 5c).

To more closely examine the kinetics of early expression of CXCL11, CXCL10 and CXCR3 in spinal cord tissue in rats immunized with MBPCFA, spinal cord tissues were collected on days 4,6 , 7 and 8 postimmunization, and RT-PCR was conducted. CXCL11 expression was not observed on day 4 or 6 postimmunization, but a low level of expression was observed on day 7 , with a further increase being observed on day 8 (Figure $5 \mathrm{~d}$ ). The expression of both CXCR3 and CXCL10 was first observed on day 6 postimmunization, with further increases observed on days 7 and 8 postimmunization. In contrast, and in keeping with the previous observations (Figure 5b), no CXCL9 expression was observed on any of these days.

\section{Expression of mRNA for CXCL11 and Related Molecules During Passive EAE}

Expression of these four genes was also examined in spinal cord tissue taken from rats into which encephalitogenic $\mathrm{T}$ lymphocytes had been transferred. EAE characteristically occurs more quickly in this passive model than in the active disease model studied in the previous group of experiments (Figure 6a). CXCL11 expression was variably detected only at a very low level on day 2 post-transfer of $\mathrm{T}$ lymphocytes, but increased on days 4 and 6 post-transfer (Figure 6b). CXCR3 expression followed a similar pattern, although expression on day 2 was clearly stronger than that observed for CXCL11 at the same point in time. In contrast to that observed for CXCL11 and similarly to that observed for CXCR3, CXCL10 expression was strong by day 2 post-transfer and continued to increase in intensity up to day 6. As observed with active EAE, CXCL9 expression in the spinal cord was very weak.

\section{Expression of CXCL11 in the Spinal Cord During Active EAE}

To determine the distribution of CXCL11 protein and its cellular source in the CNS, immunohisto-

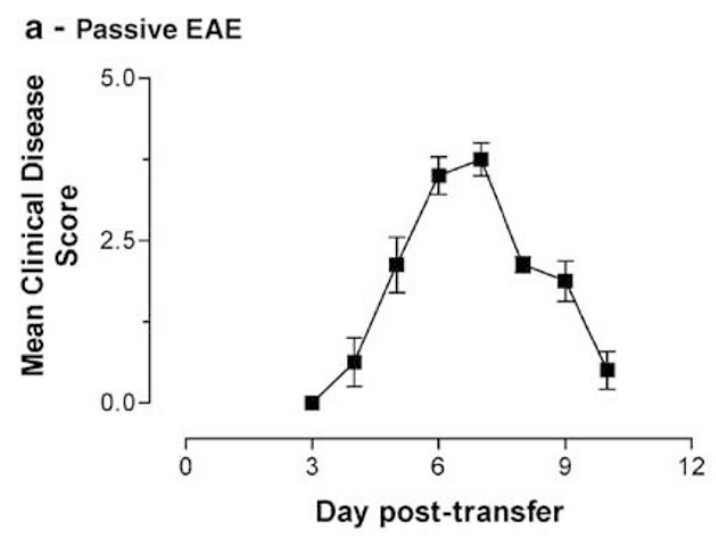

b

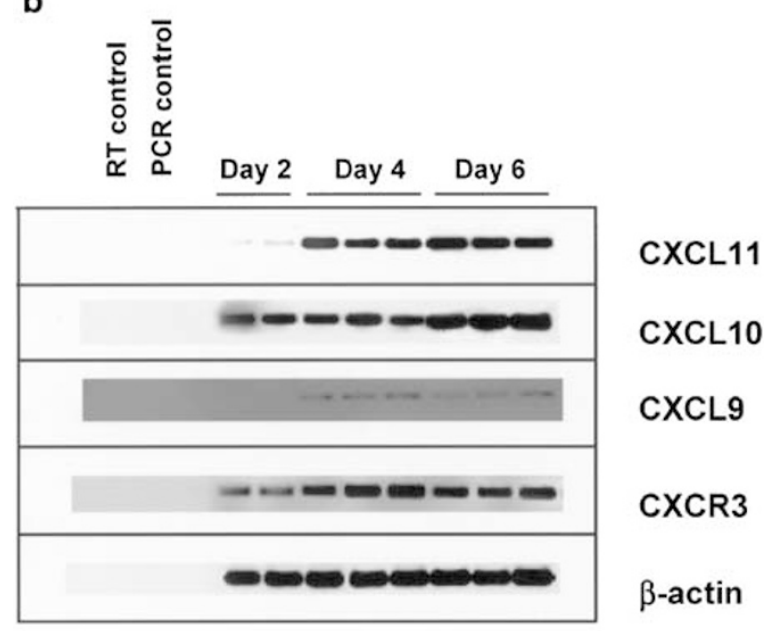

Figure 6 Expression of CXCL11 mRNA in spinal cord tissues during passive EAE. Encephalitogenic T lymphocytes were transferred into naïve recipients as described in Materials and methods and monitored for clinical signs of EAE (a). At various time points postimmunization, the animals were killed, perfused and the spinal cords collected. Total RNA was purified from the spinal cord collected and reverse-transcribed. The resultant cDNA was used as a template for PCR using oligonucleotide primers designed to amplify CXCL11, CXCL10, CXCR3 or CXCL9 (b). As a control for starting levels of template, $\beta$-actin primers were used. The data points in this figure (ie each band) are from individual rats from the same experiment. They are representative of three experiments conducted with similar results.

chemistry on serial sections of spinal cords removed from rats on day 12 postimmunization was performed. A representative example is shown in Figure 7. Typically, a mononuclear cell infiltrate was observed to form a perivascular cuff surrounding blood vessels (Figure 7a). The area immediately surrounding the blood vessels, including the parenchyma was found to stain positively with antiratCXCL11 antibodies (Figure 7b). When serial sections were stained with either ED1 to identify mononuclear phagocytes (Figure $7 \mathrm{~d}$ ) or anti-GFAP to identify astrocytes (Figure 7e), the pattern of distribution indicated that the CXCL11 distribution closely mirrored that of astrocytes (shown at higher magnification in Figure 7c, f). 

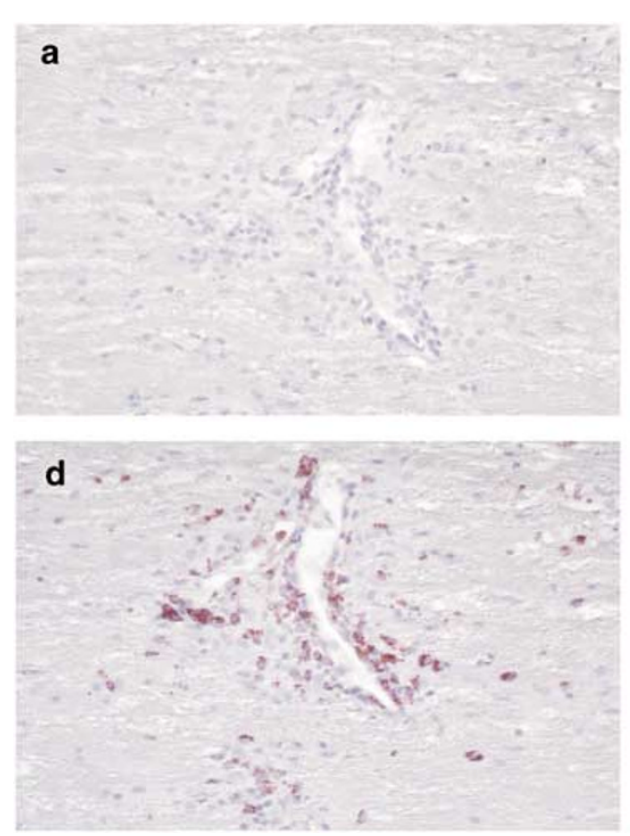
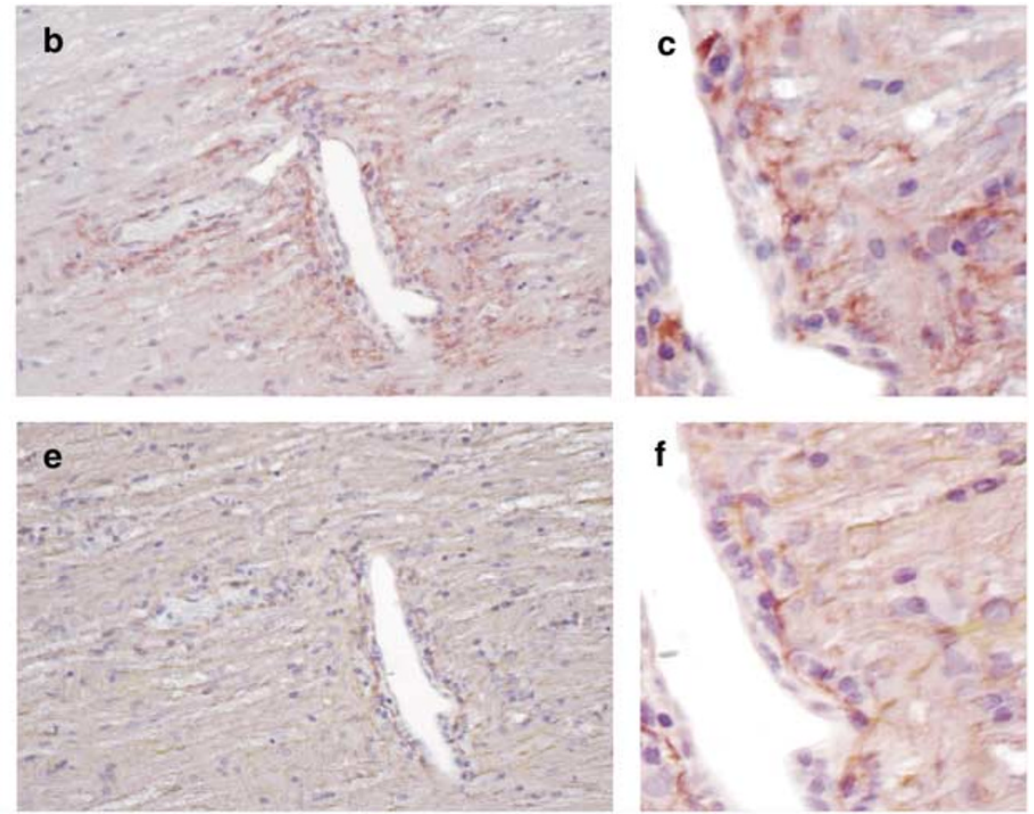

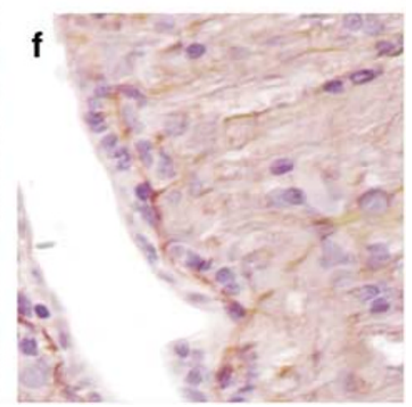

Figure 7 Expression of CXCL11 protein in the CNS during EAE. Serial paraffin sections of the lumbar spinal cord of Lewis rats with clinical EAE were subjected to antigen retrieval followed by immunohistochemistry. (a) Mayer's haematoxylin staining of an inflammatory lesion in the lumbar spinal cord of a Lewis rat with an EAE score of 5, showing numerous inflammatory cells; (b) staining using anti-rabbit ratCXCL11 IgG; (c) b at higher magnification- $-\times 40$; (d) staining using ED-1 (macrophages); (e) staining using anti-GFAP antibodies (astrocytes); (f) $\mathbf{e}$ at higher magnification $-\times 40$.

\section{Discussion}

Human CXCL11 was discovered by an EST genomics approach in IFN $\gamma$-, IL-1 $\beta$ - and TNF $\alpha$-activated astrocytes. ${ }^{13}$ Further studies showed that incubation of primary human astrocytes with a combination of IFN $\gamma$ and IL-1 $\beta$ led to a greater than 400000 -fold increase in the level of expression of CXCL11 mRNA, suggesting an important role for this chemokine in CNS inflammation that is Th1-dependent. ${ }^{13}$ However, the function and pattern of expression of this chemokine in vivo is virtually unknown. To examine the expression of CXCL11 in $\mathrm{EAE}$ in the rat, a Th1-dependent model of CNS inflammation, we cloned and characterized the rat ortholog of CXCL11.

Our data show that rat CXCL11 shares high homology at the nucleotide and amino-acid levels with human and murine CXCL11 ${ }^{13,32}$ at values that are in keeping with homologies reported for other chemokines. Based on its predicted amino-acid sequence, rat CXCL11 was synthesized and shown to activate calcium mobilization in a CXCR3expressing cell line (data not shown). It also crossdesensitized CXCR3 to another CXCR3 ligand, CXCL10, further evidence for its interaction with CXCR3. In addition, rat CXCL11 potently induced chemotaxis in vitro of rat $\mathrm{T}$ lymphocytes that had been cultured in the presence of known T-cell mitogens, including ConA, and migration of ConAactivated $\mathrm{T}$ lymphocytes from the peripheral blood into subcutaneous tissue.
To assess expression of the CXCL11 gene during the pathogenesis of EAE, oligonucleotides based on the cloned sequence for rat CXCL11 were designed and used in RT-PCR. In addition, oligonucleotides were designed from a partial rat CXCR3 cDNA clone, and primers were also designed for CXCL10 and CXCL9 based on published sequences, to allow a comparison of the expression of these three CXCR3 ligands. In actively induced disease, an increase in the level of mRNA for all three chemokines and their receptor was detected in the draining lymph nodes as early as day 2 after immunization. However, there were differences in the temporal pattern of expression of the three ligands, as well as in the intensity of the level of mRNA detected-CXCL10 expression was highest and peaked earliest, CXCL9 expression was more intense that CXCL11, but was more stable. Our data contrast somewhat to those of Narumi et $a l,{ }^{33}$ who showed little or no expression of CXCL11 in the draining lymph nodes in a similar model. However, it is possible that our ability to detect CXCL11 expression is due to the fact that we used rat CXCL11 reagents, whereas in that previous study, a mouse cDNA was used in the Northern analysis of the draining lymph nodes. The expression of CXCL10 and CXCL9 was also more intense in our study; however, this may also be due to the fact that we used a more sensitive RT-PCR/Southern blot technique compared with Northern blot analysis used by Narumi et al. These observed differences may be due to differences in the EAE model employed-in our study, purified guinea-pig MBP 
was used as an encephalitogen rather than the guinea-pig brain homogenate used in the study of Narumi et al, and it has been well established that expression and involvement of chemokines in EAE is dependent on variables such as strain of animal and antigen. ${ }^{34}$

In the spinal cord of rats with either actively or passively induced EAE, mRNA for CXCL10 and CXCR3 appeared simultaneously, around days 6 and 2 for active and passive disease, respectively. CXCL11 expression was detected later than CXCL10 expression (around days 7 and 4 for active and passive disease respectively) and CXCL9 expression was either very weak or absent. These data are again in contrast to those of Narumi et al, who showed roughly equivalent expression of CXCL10 and CXCL11 (intensity and temporal pattern) in spinal cord tissue from actively induced EAE, but it must be stated that more time points were investigated in the present study and this allowed us to determine that CXCL10 expression was clearly upregulated earlier than that of CXCL11.

The observation of simultaneous expression of both CXCL10 and CXCR3 in the present study in the spinal cords of both active and passive EAE suggests a causal role for CXCL10 in EAE. In terms of passive EAE, a recent study by Fife et $a l,{ }^{35}$ in the mouse, demonstrated the appearance of CXCL10 in CNS tissue prior to onset of clinical disease. They also demonstrated the appearance of CXCR3 on inflammatory cells infiltrating the CNS at peak disease and that neutralization of CXCL10 inhibited passive EAE. Our data therefore support the interpretation of Fife et $a,^{35}$ that the temporal expression of CXCL10 helps govern the accumulation of activated encephalitogenic $\mathrm{T}$ lymphocytes into the CNS. However, the role of CXCL10 in EAE is clearly more complex as administration of neutralizing anti-rat IP-10 (CXCL10) to female lewis rats exacerbated actively induced EAE. ${ }^{33}$ Although it is difficult to reconcile these two contrasting observations, it may be related to the quite different dosing regimens used in those two studies.

In our study on MBP-induced EAE in rats, CXCL11 mRNA was expressed at least $24 \mathrm{~h}$ after the appearance of its receptor, CXCR3, in the CNS. This strongly suggests that CXCL11 is not essential for the initial recruitment of $\mathrm{CXCR}^{+} \mathrm{T}$ cells. However, it does not rule out a possible role for CXCL11 in subsequent migration of leukocytes within the parenchyma of the CNS. The migration of neuroantigen-specific $\mathrm{T}$ cells with an activated phenotype into the CNS is orchestrated by a range of molecules including adhesion molecules, ${ }^{36,37}$ enzymes $^{38,39}$ cytokines $^{37}$ and chemokines. ${ }^{34,40}$ These cells accumulate in the perivascular space where they set up the recruitment of nonantigenspecific mononuclear cells via cytokine production. Such cytokines include IFN $\gamma$ that may also induce the production of CXCL11 by astrocytes. ${ }^{13}$ This could then enhance migration of autoreactive
$\mathrm{T}$ cells into the parenchyma where demyelination occurs.

The source of CXCL11 and other CXCR3 ligands in the CNS during the development of EAE is an important issue. These chemokines could be produced by resident cells of the CNS following activation and/or by infiltrating leukocytes including encephalitogenic $\mathrm{T}$ lymphocytes. Our data examining expression of CXCL11, CXCL10 and CXCL9 in encephalitogenic T lymphocytes cultured in vitro indicate that these cells are not a significant source of CXCR3 ligands in the CNS. Rather, resident cells are likely to be the most important source of CXCL11 and CXCL10. From previous studies, it appears that astrocytes, endothelial cells, and/or infiltrating macrophages play a prominent role in this regard: human CXCL11 was originally cloned from astrocytes ${ }^{13}$ and CXCL11 expression has been observed in cultured microglia ${ }^{13}$ and endothelial cells. ${ }^{41}$ CXCL10 expression has also been observed in these cell types ${ }^{22,41-43}$ In addition, cultured human monocytes and murine macrophages have also been shown to express CXCL11 and CXCL10 mRNA, ${ }^{32,41}$ suggesting that macrophages of peripheral blood origin infiltrating the CNS may also contribute to the expression of these chemokines during EAE. However, our observations, using immunohistochemistry suggest that astrocytes and not mononuclear phagocytes or microglia are the major source of CXCL11 during EAE and that CXCL11 expression at the protein level may be limited to astrocytes surrounding microvessels.

The issue of why three ligands exist for CXCR3 is central to our understanding of the biology of the CXCR3/ligand system. While this is not a unique occurrence in the chemokine system (other receptors also bind multiple ligands), ${ }^{5,6}$ it is particularly intriguing in this case when considering that all three ligands are expressed in response to at least one common signal-IFN $\gamma$. While there is currently no proven theory to explain this apparent redundancy, there are two main plausible explanations. First, it is possible that the three ligands are expressed in temporally and site-specific manners that are sufficiently distinct to prevent redundancy in vivo. This has been shown in subcutaneous tissue. ${ }^{29,30}$ Second, it is possible that the ligands have evolved in such a way that they bind distinctly to CXCR3 in different receptor activation states, again circumventing redundancy. A study examining binding characteristics of CXCL11 and CXCL10 indicates that these chemokines are noncompetitive or allotropic agonists for CXCR3.$^{44}$ CXCL10 exhibits a single binding affinity for CXCR3, whereas CXCL11 exhibits multiple binding affinities. The binding of these two ligands to different activation states of the receptor also differs. These data imply that ligand hierarchy exists on cell types that display different receptor conformations rasing the possibility that cells in different states of 
activation and hence bearing CXCR3 in different conformations, may vary in their sensitivity to the ligands.

In conclusion, we have cloned and characterized a rat ortholog of CXCL11, examined the expression of this gene in EAE in the rat, and compared its expression with that of its receptor, CXCR3, and two other CXCR3 ligands, CXCL10 and CXCL9. Our data clearly show an association between CXCL11 expression and T-lymphocyte-dependent CNS inflammation/pathology and although further studies using either specific neutralizing anti-CXCL11 antibodies, or CXCL11-deficient mice will be required to determine whether CXCL11 plays a causal or regulatory role in the pathogenesis of EAE, the cloning of rat CXCL11 will allow further investigation of both the biological and pathological roles of I-TAC and CXCR3.

\section{Acknowledgements}

The RNA used to clone rat CXCL11 and CXCR3 was generated by B Sahagan. We also thank Jim Boyd for synthesizing rat I-TAC. This study was supported by a grant from the National Multiple Sclerosis Society.

\section{References}

1 Janeway CA, Travers P. Immunobiology. The immune system in health and disease. Churchill Livingstone: London, 1997.

2 Butcher EC, Picker LJ. Lymphocyte homing and homeostasis. Science 1996;272:60-66.

3 Baggiolini M, Dewald B, Moser B. Human chemokines: an update. Ann Rev Immunol 1997;15:675-705.

4 Baggiolini M. Chemokines and leukocyte traffic. Nature 1998;392:565-568.

5 Gale LM, McColl SR. Chemokines: extracellular messengers for all occasions? Bioessays 1999;21:17-28.

6 Oppenheim JJ, Zachariae CO, Mukaida N, et al. Properties of the novel proinflammatory supergene 'intercrine' cytokine family. Ann Rev Immunol 1991;9: 617-648.

7 Schall TJ, Bacon KB. Chemokines, leukocyte trafficking, and inflammation. Curr Opin Immunol 1994;6: 865-873.

8 Cocchi F, DeVico A, Garzino-Demo A, et al. Identification of RANTES, MIP- $1 \alpha$ and MIP- $1 \beta$ as the major HIV suppressive factors secreted by $\mathrm{CD}^{+} \mathrm{T}$ cells. Science 1995;270:1811-1815.

9 Dragic T, Litwin V, Allaway GP, et al. HIV-1 entry into CD4+ cells is mediated by the chemokine receptor CC-CKR-5 [see comments]. Nature 1996;381:667-673.

10 Feng Y, Broder CC, Kennedy PE, et al. HIV-1 entry cofactor: functional cDNA cloning of a seven-transmembrane, G protein-coupled receptor. Science 1996; 272:872-877.

11 Zimmerman PA, Buckler White A, Alkhatib G, et al. Inherited resistance to HIV-1 conferred by an inactivating mutation in CC chemokine receptor 5: studies in populations with contrasting clinical phenotypes, defined racial background, and quantified risk. Mol Med 1997;3:23-36.

12 Zlotnik A, Yoshie O. Chemokines: a new classification system and their role in immunity. Immunity 2000;12: 121-127.

13 Cole KE, Strick CA, Paradis TJ, et al. Interferoninducible T cell alpha chemoattractant (I-TAC): a novel non-ELR CXC chemokine with potent activity on activated T cells through selective high affinity binding to CXCR3. J Exp Med 1998;187:2009-2021.

14 Luster AD, Ravetch JV. Biochemical characterization of a gamma interferon-inducible cytokine (IP-10). J Exp Med 1987;166:1084-1097.

15 Farber JM. A macrophage mRNA selectively induced by gamma-interferon encodes a member of the platelet factor 4 family of cytokines. Proc Natl Acad Sci USA 1990;87:5238-5242.

16 Loetscher M, Gerber B, Loetscher P, et al. Chemokine receptor specific for IP10 and mig: structure, function, and expression in activated T-lymphocytes. J Exp Med 1996;184:963-969.

17 Bonecchi R, Bianchi G, Bordignon PP, et al. Differential expression of chemokine receptors and chemotactic responsiveness of type $1 \mathrm{~T}$ helper cells (Th1s) and Th2s. J Exp Med 1998;187:129-134.

18 Loetscher M, Gerber B, Loetscher P, et al. Chemokine receptor specific for IP10 and mig: structure, function, and expression in activated T-lymphocytes. J Exp Med 1996;184:963-969.

19 Sallusto F, Lenig D, Mackay CR, et al. Flexible programs of chemokine receptor expression on human polarized T helper 1 and 2 lymphocytes. J Exp Med 1998;187:875-883.

20 Ebert LM, Caon AC, Clark-Lewis I, et al. Coregulation of chemokine receptors and CD4 during allogeneic activation of $\mathrm{T}$ lymphocytes. J Immunol 2001;166: 4870-4878.

21 Arimilli S, Ferlin W, Solvason N, et al. Chemokines in autoimmune diseases. Immunol Rev 2000;177: 43-51.

22 Balashov KE, Rottman JB, Weiner HL, et al. CCR5(+) and CXCR3(+) $\mathrm{T}$ cells are increased in multiple sclerosis and their ligands MIP-1alpha and IP-10 are expressed in demyelinating brain lesions. Proc Natl Acad Sci USA 1999;96:6873-6878.

23 Rottman JB, Smith TL, Ganley KG, et al. Potential role of the chemokine receptors CXCR3, CCR4, and the integrin alphaEbeta7 in the pathogenesis of psoriasis vulgaris. Lab Invest 2001;81:335-347.

24 Patel DD, Zachariah JP, Whichard LP. CXCR3 and CCR5 ligands in rheumatoid arthritis synovium. Clin Immunol 2001;98:39-45.

25 Katschke Jr KJ, Rottman JB, Ruth JH, et al. Differential expression of chemokine receptors on peripheral blood, synovial fluid, and synovial tissue monocytes/ macrophages in rheumatoid arthritis. Arthritis Rheum 2001;44:1022-1032.

26 Sauty A, Dziejman M, Taha RA, et al. The T cellspecific CXC chemokines IP-10, Mig, and I-TAC are expressed by activated human bronchial epithelial cells. J Immunol 1999;162:3549-3558.

27 Altschul SF, Gish W, Miller W, et al. Basic local alignment search tool. J Mol Biol 1990;215: 403-410.

28 Nelson RT, Boyd J, Gladue RP, et al. Genomic characterization of the CC chemokine macrophage inflammatory protien- $3 \alpha$ (MIP- $3 \alpha)$, showing gene 
structure, splice variants and chromosome localization. Genomics 2001;73:28-37.

29 Tessier PA, Naccache PH, Diener KR, et al. Induction of acute inflammation in vivo by staphylococcal superantigens. II. Critical role for chemokines, ICAM-1, and TNF-alpha. J Immunol 1998;161: 1204-1211.

30 Tessier PA, Naccache PH, Clark-Lewis I, et al. Chemokine networks in vivo: involvement of $\mathrm{C}-\mathrm{X}-\mathrm{C}$ and $\mathrm{C}-\mathrm{C}$ chemokines in neutrophil extravasation in vivo in response to TNF-alpha. J Immunol 1997;159: 3595-3602.

31 Ben-Nun A, Wekerle H, Cohen IR. The rapid isolation of clonable antigen-specific $\mathrm{T}$ lymphocyte lines capable of mediating experimental allergic encephalomyelitis. Eur J Immunol 1981;25:1-6.

32 Widney DP, Xia YR, Lusis AJ, et al. The murine chemokine CXCL11 (IFN-inducible $\mathrm{T}$ cell alpha chemoattractant) is an IFN-gamma- and lipopolysaccharide-inducible glucocorticoid-attenuated response gene expressed in lung and other tissues during endotoxemia. J Immunol 2000;164:63226331.

33 Narumi S, Kaburaki T, Yoneyama H, et al. Neutralization of IFN-inducible protein 10/CXCL10 exacerbates experimental autoimmune encephalomyelitis. Eur J Immunol 2002;32:1784-1791.

34 Karpus WJ, Ransohoff RM. Chemokine regulation of experimental autoimmune encephalomyelitis: temporal and spatial expression patterns govern disease pathogenesis. J Immunol 1998;161:2667-2671.

35 Fife BT, Kennedy KJ, Paniagua MC, et al. CXCL10 (IFN-gamma-inducible protein-10) control of encephalitogenic CD4+ $\mathrm{T}$ cell accumulation in the central nervous system during experimental autoimmune encephalomyelitis. J Immunol 2001;166: $7617-7624$.
36 Baron JL, Madri JA, Ruddle NH, et al. Surface expression of alpha 4 integrin by CD4T cells is required for their entry into brain parenchyma. J Exp Med 1993;177:57-68.

37 Kuchroo VK, Martin CA, Greer JM, et al. Cytokines and adhesion molecules contribute to the ability of myelin proteolipid protein-specific $\mathrm{T}$ cell clones to mediate experimental allergic encephalomyelitis. J Immunol 1993;151:4371-4382.

38 Willenborg DO, Parish CR, Cowden WB. Phosphosugars are potent inhibitors of central nervous system inflammation. Faseb J 1989;3:1968-1971.

39 Yong VW, Chabot S, Stuve O, et al. Interferon beta in the treatment of multiple sclerosis: mechanisms of action. Neurology 1998;51:682-689.

40 Karpus WJ, Kennedy KJ, Fife BT, et al. Chemokine regulation of immune-mediated demyelinating disease. Ilar J 1999;40:183-189.

41 Mach F, Sauty A, Iarossi AS, et al. Differential expression of three $\mathrm{T}$ lymphocyte-activating $\mathrm{CXC}$ chemokines by human atheroma-associated cells. J Clin Invest 1999;104:1041-1050.

42 Hua LL, Lee SC. Distinct patterns of stimulusinducible chemokine mRNA accumulation in human fetal astrocytes and microglia. Glia 2000;30: 74-81.

43 Ren LQ, Gourmala N, Boddeke HW, et al. Lipopolysaccharide-induced expression of IP-10 mRNA in rat brain and in cultured rat astrocytes and microglia. Brain Res Mol Brain Res 1998;59: 256-263.

44 Cox MA, Jenh CH, Gonsiorek W, et al. Human interferon-inducible $10-\mathrm{kDa}$ protein and human interferon-inducible $\mathrm{T}$ cell alpha chemoattractant are allotopic ligands for human CXCR3: differential binding to receptor states. Mol Pharmacol 2001;59: 707-715 